TIFR-TH-05-34

hep-th/0512005

\title{
Generalised T-Duality and Non-Geometric Backgrounds
}

\author{
Atish Dabholkar a and Chris Hull ${ }^{\mathrm{b}}$ \\ ${ }^{a}$ Department of Theoretical Physics, Tata Institute of Fundamental Research, \\ Homi Bhabha Road, Mumbai 400005, India \\ b The Blackett Laboratory, Imperial College London \\ Prince Consort Road, London SWr 2AZ, United Kingdom \\ b The Institute for Mathematical Sciences, Imperial College London, \\ 48 Princes Gardens, London SW' 2AZ, United Kingdom
}

\begin{abstract}
We undertake a systematic analysis of non-geometric backgrounds in string theory by seeking stringy liftings of a class of gauged supergravity theories. In addition to conventional flux compactifications and non-geometric T-folds with T-duality transition functions, we find a new class of non-geometric backgrounds with non-trivial dependence on the dual coordinates that are conjugate to the string winding number. We argue that T-duality acts in our class of theories, including those cases without isometries in which the conventional Buscher rules cannot be applied, and that these generalised T-dualities can take T-folds or flux compactifications on twisted tori to examples of the new non-geometric backgrounds. We show that the new class of non-geometric backgrounds and the generalised T-dualities arise naturally in string field theory, and are readily formulated in terms of a doubled geometry, related to generalised geometry. At special points in moduli space, some of the non-geometric constructions become equivalent to asymmetric orbifolds which are known to provide consistent string backgrounds. We construct the bosonic sector of the corresponding gauged supergravity theories and show that they have a universal form in any dimension, and in particular construct the scalar potential. We apply this to the supersymmetric WZW model, giving the complete non-linear structure for a class of WZW-model deformations.
\end{abstract}

Email: atish@tifr.res.in, c.hull@imperial.ac.uk 


\section{Introduction}

Superstring theory can be formulated in 'non-geometric' backgrounds that cannot be understood in terms of supergravity and conventional field theory [1, 2, 3, 4, 5, 6, 7, 8, 9, and these offer an interesting window onto stringy physics beyond the scope of supergravity. The purpose of this paper is to systematically analyse the possible structures of non-geometric backgrounds and seek string-theory constructions of these.

The non-geometric backgrounds that have been studied so far are backgrounds in which the transition functions are allowed to include duality transformations, and a systematic study of those with T-duality transition functions was made in [1, where the name Tfold was proposed. Our considerations will lead us to a new class of backgrounds that are even more non-geometric than T-folds: whereas T-folds look like conventional spacetimes locally, with ordinary spacetime patches glued together with transition functions that can include T-dualities, the new class of backgrounds do not look like conventional spaces even locally. Nonetheless, we shall present arguments that these non-geometric spaces are good string backgrounds and that they necessarily arise in string theory. In some cases, these new backgrounds are obtained from consistent backgrounds by the action of T-dualities. However, these are T-dualities in directions in which there are no isometries, so that the conventional sigma-model duality transformations through the Buscher rules cannot be applied. We argue that T-duality can nonetheless be applied, with a more general set of rules.

A classic example is that of a three-torus with constant $H$-flux, so that the NS-NS 3 form field strength is proportional to the volume form on $T^{3}$. Performing one T-duality gives a $T^{2}$ bundle over $S^{1}[10$ with monodromy in the group $S L(2, \mathbb{Z})$ of large diffeomorphisms on $T^{2}$. A further T-duality on one of the fibre directions gives a non-geometric T-fold that is a $T^{2}$ bundle over $S^{1}$, but with monodromy in the T-duality group $O(2,2 ; \mathbb{Z})$ [4, [6], , so that this should be thought of as a bundle of CFTs over a circle with each fibre being a CFT on $T^{2}$ and the moduli of these CFTs varying over the $S^{1}$. A further T-duality on the $S^{1}$ base appears impossible as the T-fold geometry depends explicitly on the circle coordinate. We propose a construction of the missing T-dual.

This has interesting applications to mirror symmetry on Calabi-Yau manifolds with flux. Consider a CY with $T^{3}$ fibration with $H$-flux on the fibres. If the argument of [1] generalises to the case with flux, then one expects the mirror background in the large volume limit to be obtained by acting with T-dualities on all fibre directions. In this case, the T-dual must be a non-geometric background of the type we propose here.

We show that the new non-geometric backgrounds and the generalised T-dualities arise naturally in string field theory, and indeed generic solutions of string field theory will be non-geometric backgrounds of this type. We also show that in some cases the new exotic

backgrounds have a point in moduli space that minimises a scalar potential and at which 
the theory can be constructed by a special type of asymmetric orbifold, so that our new constructions can be thought of as giving deformations of asymmetric orbifolds.

The new backgrounds arise naturally in the doubled formalism used in [1. Strings on a torus $T^{n}$ can be formulated on a doubled torus $\bar{T}^{2 n}$ with $n$ coordinates $X^{i}$ conjugate to the momenta and $n$ coordinates $\tilde{X}^{i}$ conjugate to the winding modes. Geometric backgrounds and T-folds can have non-trivial dependence on the space coordinates $X^{i}$ in the usual way, while our new backgrounds have non-trivial dependence on $\tilde{X}$ also. For example, a $T^{n}$ bundle over $T^{m}$ gives a geometric background, a $\bar{T}^{2 n}$ bundle over $T^{m}$ gives a $\mathrm{T}$-fold while bundles of the doubled torus $\bar{T}^{2 n}$ over the doubled base $\bar{T}^{2 m}$ give examples of our new class of non-geometric backgrounds. Indeed, the T-dual of the $T^{3}$ with $H$-flux is a bundle of this kind with $n=2, m=1$.

Our approach here is to investigate the lifting of a certain class of $D$-dimensional gauged supergravities to 10 dimensions. In some cases they arise from supergravity compactifications, but for others there is no such geometric origin. Some arise from intrinsically stringy constructions such as asymmetric orbifolds, reductions with duality twists or T-fold reductions. In general, the lifting is non-geometric in the sense that there is no supergravity compactification that gives rise to them. For example, compactification on a manifold with an isometry group $K$ gives a theory in which the vector fields from the reduction of the metric are gauge fields for a Yang-Mills group $K$, but the gauge fields arising from the reduction of antisymmetric tensor gauge fields typically have abelian self-interactions, so that the gauge group for the reduction of a theory with gravity plus anti-symmetric tensors is a product or semi-direct product of $K$ with an abelian group. There are gauged supergravities in which the $D$-dimensional gauge fields that are associated with antisymmetric tensor gauge fields become non-abelian in $D$ dimensions, but such non-abelian interactions cannot arise from a conventional compactification. Roughly speaking, the curving of the internal geometry leading to an isometry group gives a non-abelian structure to the momentum modes, and what would be needed is the dual version of this that gives a non-abelian structure to string winding modes or brane wrapping modes, and this is clearly beyond the scope of supergravity. The non-geometric background we find gives the required 'curving' of the dimensions conjugate to the string winding modes

An important question is whether all effective supergravity theories have a stringy lifting. We find classical stringy liftings for a wide class of 'unliftable' supergravities and we have found no obstructions to finding such lifitings generally. However, in this paper we consider only classical string theory, and at the quantum level modular invariance is expected to restrict the possible non-geometric backgrounds, just as it does for asymmetric orbifolds. However, those that arise as T-duals of consistent backgrounds should be quantum consistent.

We focus here on the sector associated with the metric, B-field and dilaton, and on the action of T-duality, as we are interested here in explicit string theory constructions, but 
this can be generalised to include U-duality and RR or heterotic sectors. The dimensional reduction of this sector on a $d$-torus and truncation to a particular finite subset of fields gives a theory with an $O(d, d)$ symmetry, $2 d$ vector fields and $d^{2}+1$ scalars. The gauged theories we consider are ones in which a $2 d$-dimensional subgroup of $O(d, d)$ is promoted to a local symmetry with the spin one fields becoming the gauge fields. We argue that the general gauged supergravities must be of the same form as the ones found by Kaloper and Myers 12. for Scherk-Schwarz reductions with flux, generalised to a wider class of gauge groups than considered there. In particular, we find the form of the scalar potential for the $d^{2}+1$ scalars.

The effective $D$-dimensional supergravity theories that we consider give important information about the structure of the 10-dimensional liftings, when they exist, and provide a way of parameterising the possible backgrounds that might emerge. In general, these arise from a (possibly non-geometric) compactification followed by a truncation. There is a natural action of $O(d, d)$ on the effective supergravity, which takes it to an apparently different but physically equivalent $D$-dimensional theory. However, these transformations can have radical effects on the 10-dimensional liftings, for example taking one from a geometric compactification in which the non-abelian gauge fields arise from isometries to a non-geometric one in which the non-abelian vector fields come from the $B$-fields coupling to winding modes. This gives an indication as to how T-duality must act in the uplifted theory. The scalar potential also gives important information about the structure of the theories. Of particular interest are critical points of the scalar potential with zero energy, as they correspond to reductions to $D$-dimensional Minkowski space. An important class of these arise from asymmetric orbifold constructions, so that the non-geometric deformations away from the orbifold point in moduli space that we propose correspond to moving away from the minimum of the potential, and there will in general no longer be a solution involving $D$-dimensional Minkowski space. In these cases, the solution of the $D$-dimensional effective theory which has maximal symmetry is typically a solution of domain-wall type with $D-1$ dimensional Poincaré symmetry.

The plan of this paper is as follows. In $\$ 2$ we review the low energy effective action for toroidal compactifications of string theory and consider the most general gaugings. ScherkSchwarz reductions and duality-twisted reductions are special cases of these. Based on the known formula for the effective potential that arises in this case, we propose an effective potential for the most general gauging based on the duality covariance of the formula and discuss some properties of the general gauge algebra in \$3. We review and discuss ScherkSchwarz reductions and duality-twisted reductions in sections $\$ 4$ and $\$ 5$ respectively. In 96 , we apply our formalism to WZW models and argue that the potential proposed in 92 correctly captures the non-linear structure for a class of WZW-model deformations. In particular, the potential gives the correct symmetry breaking pattern after including an infinite number of higher order terms in the effective potential for the Higgs field that breaks the gauge 
symmetry. In $\$ 7$ we show that some of the models obtained by lifting gaugings are related by duality to known Scherk-Schwarz and duality-twisted reductions, and investigate in detail the special cases in which these reduce to asymmetric orbifold constructions which can be dualised explicitly. In $\$ 8$ we examine the new non-geometric constructions implied by the generalised T-duality. In $\$ 9$ we argue that the generalised T-duality can be implemented within string field theory and that general string field theory solutions will include nongeometric backgrounds of the type we discuss here. We conclude with a discussion in \$10.

While this paper was in preparation, the paper [8] appeared in which the relation between low-energy effective actions in four dimensions and non-geometric backgrounds were also explored. They also identified classes of gaugings that might lead to new non-geometric backgrounds, but no constructions of such backgrounds were provided.

\section{The Low-Energy Effective Action and Gauged Su- pergravity}

The low-energy effective field theory for $n$-dimensional string theory includes the terms

$$
S=\int d^{n} x \sqrt{-\mathcal{G}} e^{-\Phi}\left\{\mathcal{R}+(\nabla \Phi)^{2}-\frac{1}{12} \mathcal{H}_{\mu \nu \lambda} \mathcal{H}^{\mu \nu \lambda}\right\}
$$

for a scalar field $\Phi$, metric $\mathcal{G}_{\mu \nu}$ and 2-form gauge field $\mathcal{B}$ with field strength $\mathcal{H}=d \mathcal{B}$. Our conventions are as in [12, and our notation largely follows that of [12. This action can arise as part of the low-energy effective action of the bosonic string with $n=26$ or of the 'common sector' of the heterotic or type II superstrings in $n=10$ dimensions. The 10dimensional supergravity theories describing the low-energy limit of the heterotic or type II string theories can be decomposed into $D=10, N=1$ multiplets. In all cases, there is a common sector consisting of the $N=1$ supergravity multiplet whose bosonic fields consist of the metric, the 2-form gauge field $\mathcal{B}$ and the dilaton, with action (2.1). For the heterotic or type I strings, there are in addition Yang-Mills multiplets, while for the type II strings one adds a gravitino multiplet whose highest spin state is a gravitino, and there are two possibilities depending on whether this has the same or the opposite chirality to the gravitino in the supergravity multiplet. We will discuss in this paper the reduction of the sector of the low-energy theory represented by the action (2.1), corresponding to the $N=1$ supergravity sector of the theory, obtaining a gauged supergravity with 16 supersymmetries that is a truncation of the gauged supergravity arising from the full theory. The reduction of the full heterotic theory is discussed in [12, while that of the type II theory will be discussed in [13. In particular, the scalar potential we obtain will be restricted to the scalars coming from the reduction of the common sector. However, in the full theory there are other scalars

coming from the RR fields in the type II string and from the Yang-Mills gauge fields in the 
heterotic string, and the full potential depends on these also. The gauge symmetry of the full theory will contain the gauge algebra that we discuss in section 3, but will be larger in general. The generalisation to M-theory will be discussed in [13.

The standard Kaluza-Klein dimensional reduction of (2.1) on a $d$-torus gives a theory in $D=n-d$ dimensions with an $O(d, d)$ invariance and action [14, 12]

$$
\begin{aligned}
S=\int d^{D} x \sqrt{-g} e^{-\phi} & \left\{R+(\nabla \phi)^{2}-\frac{1}{12} H_{\mu \nu \lambda}^{2}\right. \\
+ & \left.\frac{1}{8} L_{a b} \nabla_{\mu} \mathcal{K}^{b c} L_{c d} \nabla^{\mu} \mathcal{K}^{d a}-\frac{1}{4} F_{\mu \nu}^{a} L_{a b} \mathcal{K}^{b c} L_{c d} F^{d \mu \nu}\right\}
\end{aligned}
$$

The $d$ Kaluza-Klein vector fields $V^{M}{ }_{\mu}(M, N=1, \ldots, d)$ from the reduction of the metric and the $d$ vector fields $B_{\mu M}$ from the reduction of $\mathcal{B}$ have been combined into the $2 d$ vector fields $A_{\mu}^{a}(a, b=1, \ldots, 2 d)$ with abelian field strengths $F^{a}=d A^{a}$

$$
A_{\mu}^{a}=\left(\begin{array}{l}
V_{\mu}^{M} \\
B_{\mu M}
\end{array}\right)
$$

These vector fields transform as the fundamental representation of $O(d, d), A^{a} \rightarrow A^{\prime a}=$ $M_{b}^{a} A^{b}$. The $O(d, d)$ invariant metric is

$$
L^{a b}=\left(\begin{array}{ll}
0 & \mathbf{1} \\
\mathbf{1} & 0
\end{array}\right)
$$

where 1 is the $d \times d$ unit matrix, and satisfies $M L M^{T}=L$, where the superscript $T$ indicates matrix transposition. The scalar fields take values in the coset $O(d, d) / O(d) \times O(d)$ and are represented by a symmetric $2 d \times 2 d$ matrix $\mathcal{K}^{a b}$ with trace $L_{a b} \mathcal{K}^{a b}=0$ and which transforms under $O(d, d)$ as $\mathcal{K}^{a b} \rightarrow \mathcal{K}^{\prime a b}=M^{a}{ }_{c} M^{b}{ }_{d} \mathcal{K}^{c d}$. The reduced three-form field strength is

$$
H_{\mu \nu \lambda}=\partial_{\mu} B_{\nu \lambda}-\frac{1}{2} A_{\mu}^{a} L_{a b} F_{\nu \lambda}^{b}+\text { cyclic permutations }
$$

and includes abelian Chern-Simons terms [17]. The theory has $U(1)^{2 d}$ gauge symmetry and a manifest global $O(d, d)$ symmetry.

We will be interested in gauged supergravities that arise as deformations of this theory. As we shall review in $\$ 4$, a class of these arise from dimensional reduction on twisted tori with flux. In the class of gauged supergravities that we shall consider here, a $2 d$-dimensional subgroup $G$ of $O(d, d)$ is promoted to a local symmetry, with the gauge fields $A^{a}$ appearing in the minimal couplings. For this to be possible, it is essential that the fundamental representation of $O(d, d)$ becomes the adjoint of $G$ under the embedding of $G$ in $O(d, d)$, so that the gauge fields $A^{a}$ transform in the adjoint of the gauge group. If the generators of $O(d, d)$ are denoted by $t_{a b}=-t_{b a}$, then the subgroup $G$ generators $T_{a}$ are specified by an embedding tensor $\Theta_{a}{ }^{b c}$ so that

$$
T_{a}=\frac{1}{2} \Theta_{a}^{b c} t_{b c}
$$


and satisfy

$$
\left[T_{a}, T_{b}\right]=i f_{a b}{ }^{c} T_{c}
$$

where $f_{a b}{ }^{c}$ are the structure constants of $G$.

The deformation of (2.2) with gauge symmetry $G$ is

$$
\begin{aligned}
S=\int d^{D} x \sqrt{-g} e^{-\phi}\left\{R+(\nabla \phi)^{2}+\frac{1}{8} L_{a b} \mathcal{D}_{\mu} \mathcal{K}^{b c} L_{c d} \mathcal{D}^{\mu} \mathcal{K}^{d a}\right. \\
\left.-\frac{1}{4} F_{\mu \nu}^{a} L_{a b} \mathcal{K}^{b c} L_{c d} F^{d \mu \nu}-\frac{1}{12} H_{\mu \nu \lambda}^{2}-g^{2} W(\mathcal{K})\right\}
\end{aligned}
$$

where $W(\mathcal{K})$ is a gauge-invariant scalar potential, the covariant derivative of the scalar fields is

$$
\mathcal{D}_{\mu} \mathcal{K}^{a b}=\partial_{\mu} \mathcal{K}^{a b}-g f_{c d}{ }^{a} A_{\mu}^{c} \mathcal{K}^{d b}-g f_{c d}{ }^{b} A_{\mu}^{c} \mathcal{K}^{a d} .
$$

the field strengths for the gauge fields $A$ taking values in the Lie algebra of $G$ are

$$
F=d A+i g A \wedge A=\frac{1}{2} F_{\mu \nu}^{a} T_{a} d x^{\mu} \wedge d x^{\nu}
$$

and the three-form field strength is

$$
H=d B-\frac{1}{2} \omega_{C S}
$$

where the Chern-Simons term for $G$ is

$$
\omega_{C S}=\operatorname{tr}\left(A \wedge F-g \frac{i}{3} A \wedge A \wedge A\right)
$$

Here $g$ is the gauge coupling constant, which will be set to $g=1$ in later sections. In the supersymmetric case, this is the bosonic sector of a gauged supergravity action in which the fermionic terms are modified by fermion mass terms proportional to $g$.

All the terms in the action (2.8) are completely determined by gauge invariance and requiring the ungauged limit to give (2.2), apart from the scalar potential, which in principle could be any $G$-invariant function of the scalars in the bosonic string theory. However, for the superstring, the theory resulting from the reduction of the supergravity action whose bosonic part contains (2.8) is a gauged supergravity in $D$ dimensions and supersymmetry leads to further restrictions. Supersymmetry in general rules out some of the possible gauge groups, and determines the form of the scalar potential. The minimal couplings for the class of the theories considered here only depend on the embedding tensor through the structure constants $f_{a b}{ }^{c}$, and on general grounds the supersymmetric scalar potential must be $O(d, d)$ covariant and must be constructed from the structure constants $f_{a b}{ }^{c}$, the scalar matrix $\mathcal{K}^{a b}$ and the $O(d, d)$ metric $L^{a b}$. Moreover, it must be quadratic in the structure constants and of order $g^{2}$ and so the general form must be (using the notation $f_{a b c}=f_{a b}{ }^{d} L_{c d}$, which is automatically antisymmetric $\left.f_{a b c}=f_{[a b c]}\right)$

$$
W_{\text {gen }}(\mathcal{K})=a \mathcal{K}^{a d} \mathcal{K}^{b e} \mathcal{K}^{c f} f_{a b c} f_{\text {def }}+b \mathcal{K}^{a d} \mathcal{K}^{b e} L^{c f} f_{a b c} f_{\text {def }}+c \mathcal{K}^{a d} L^{b e} L^{c f} f_{a b c} f_{\text {def }}+d
$$


for some constants $a, b, c, d$, and it must take this form with the same constants $a, b, c, d$ for all possible gaugings.

In the generalised Scherk-Schwarz reductions with flux to be discussed in $₫$, the scalar potential is given by $W(\mathcal{K})=W_{S S}(\mathcal{K})$ [12, 15] where

$$
W_{S S}(\mathcal{K})=\frac{1}{12} \mathcal{K}^{a d} \mathcal{K}^{b e} \mathcal{K}^{c f} f_{a b c} f_{d e f}-\frac{1}{4} \mathcal{K}^{a d} L^{b e} L^{c f} f_{a b c} f_{d e f}
$$

This must agree with the general potential, and is sufficiently general to fix the coefficients $a, b, c, d$, and so the potential must be $W(\mathcal{K})=W_{S S}(\mathcal{K})$ given by (2.14) for all supersymmetric gaugings. Our discussion of explicit string compactifications and their low-energy limits will provide non-trivial checks of this conclusion. Note that in general there could be quantum corrections to this potential.

The ungauged action is manifestly invariant under $O(d, d)$ transformations, while the couplings of the gauged action (2.8) break this to the subgroup preserving the structure constants $f_{a b}{ }^{c}$. However, it becomes invariant if the structure constants are also taken to transform

$$
f_{a b}^{c} \rightarrow M_{a}^{d} M_{b}^{e} M_{f}^{c} f_{d e}^{f}
$$

so that the embedding also changes.

\section{The Gauge Algebra and Duality}

As we shall review in the next section, generalised Scherk-Schwarz reduction is specified by two constant tensors, the structure constants $\gamma_{N P}^{M}$ of a Lie group $K$ specifying the 'twisting' of the basis one-forms $\eta^{M}$ in (4.1), and the constraints $\beta_{M N P}$ specifying a 3 -form flux

$$
\frac{1}{2} \beta_{M N P} \eta^{M} \wedge \eta^{N} \wedge \eta^{P}
$$

for the 3 -form field strength $\mathcal{H}$.

As we have seen, the $2 d$ gauge fields $A^{a}$ consist of $d$ gauge fields $V^{M}$ arising from the reduction of the metric and $d$ gauge fields $B_{M}$ from the reduction of the B-field. The gauge generators $T_{a}$ decompose into the generators $Z_{M}$ corresponding to $V^{M}$ and $X^{M}$ corresponding to $B_{M}$. The internal space has isometry group $K$, and in the absence of flux the gauge group is a semi-direct product of the group containing $K$ generated by $Z_{M}$ and an abelian group generated by the $X^{M}$. With flux, this is deformed to [12, 15]

$$
\begin{aligned}
& {\left[X^{M}, X^{N}\right]=0} \\
& {\left[X^{M}, Z_{N}\right]=2 i \gamma_{N P}^{M} X^{P}} \\
& {\left[Z_{M}, Z_{N}\right]=2 i \gamma_{M N}^{P} Z_{P}-3 i \beta_{M N P} X^{P}}
\end{aligned}
$$


and the low-energy effective action is given by (2.8) and (2.14) based on this algebra. The Jacobi identities require that $\gamma_{N P}^{M}$ are the structure constants of a Lie algebra $K$ and that

$$
3 \beta_{L[M N} \gamma_{P Q]}^{L}=0
$$

The most general gauge algebra will have a decomposition

$$
\begin{aligned}
& {\left[X^{M}, X^{N}\right]=2 i \tilde{\gamma}_{P N}^{M N} X^{P}+3 i \tilde{\beta}^{M N P} Z_{P}} \\
& {\left[X^{M}, Z_{N}\right]=2 i \hat{\gamma}_{N P}^{M} X^{P}+2 i \bar{\gamma}_{N}^{M P} Z_{P}} \\
& {\left[Z_{M}, Z_{N}\right]=2 i \gamma_{M N}^{P} Z_{P}-3 i \beta_{M N P} X^{P}}
\end{aligned}
$$

and is specified by a set of tensors $\gamma, \beta, \tilde{\gamma} \ldots$, subject to the constraints arising from the Jacobi identities.

These then constitute the general set of parameters specifying the low-energy field theory generalising the twist $\gamma$ and flux $\beta$ of the usual reduction. We will refer to $\tilde{\gamma}$ as the dual twist and $\tilde{\beta}$ as the dual flux. Our aim here is to find string constructions in which some of these new parameters $\tilde{\gamma}, \hat{\gamma}, \bar{\gamma}, \tilde{\beta}$ are non-trivial. In some cases, these arise from non-geometric backgrounds, but as we shall see in $₫$, they can also arise from geometric compactifications that are not of Scherk-Schwarz type.

As we saw in the last section, there is a natural action of $O(d, d)$ under which the structure constants $f_{a b}^{c}$ transform covariantly. These transformations mix the twist $\gamma$ and flux $\beta$ with each other and with the new parameters $\tilde{\gamma}, \hat{\gamma}, \bar{\gamma}, \tilde{\beta}$ in general. This gives a natural conjecture for the action of the T-duality group $O(d, d ; \mathbb{Z})$ in the full string theory [15], namely that the structure constants transform as (2.15). This reproduces the known cases in which T-duality acts through the Buscher rules [19] and interchange twist and flux (e.g. acting on a 3-torus with constant H-flux $\beta$ and $\gamma=0$ with a T-duality on one circle gives a torus bundle over a circle with twist $\gamma$ but no flux, so that $\beta=0[10]$ ). Moreover, this predicts how to extend this to non-geometric backgrounds.

\section{Scherk-Schwarz Reductions with Flux}

In this section we review the results of [16, 12, 15] for the general Scherk-Schwarz reduction with flux of the theory with action (2.1) to $D$ dimensions. The internal manifold is taken to be a space with a basis of one-forms $\eta^{M}$ satisfying

$$
d \eta^{M}=-\gamma_{N P}^{M} \eta^{N} \wedge \eta^{P}
$$

with constant coefficients $\gamma_{N P}^{M}$, so that the integrability condition for (4.1) is that the $\gamma_{N P}^{M}$ satisfy the Jacobi identity

$$
\gamma_{R[N}^{M} \gamma_{P Q]}^{R}=0
$$


and so are the structure constants of the Lie algebra for some group $K$. Note that $K$ can be non-compact or non-semi-simple. The Scherk-Schwarz reduction ansatz for tensor fields is that they have components with respect to the $\eta$-frame that are independent of the internal coordinates $y$, so that the internal metric is

$$
d s^{2}=\mathcal{G}_{M N} \eta^{M} \eta^{N}
$$

where $\mathcal{G}_{M N}$ is independent of the internal coordinates $y$. The $\mathcal{G}_{M N}$ are moduli for the internal metric and in the full ansatz can depend on the remaining coordinates $x^{\mu}$, giving rise to scalar fields $\mathcal{G}_{M N}(x)$. Similarly, the internal B-field could be taken to be $\mathcal{B}=\frac{1}{2} \mathcal{B}_{M N} \eta^{M} \wedge \eta^{N}$. This can be generalised to include a flux $\frac{1}{2} \beta_{M N P} \eta^{M} \wedge \eta^{N} \wedge \eta^{P}$ for some constants $\beta_{M N P}$. This will be a closed 3 -form if

$$
\beta_{L[M N} \gamma_{P Q]}^{L}=0
$$

Then locally there is a 2 -form $\omega$ so that

$$
d \omega=\frac{1}{2} \beta_{M N P} \eta^{M} \wedge \eta^{N} \wedge \eta^{P}
$$

and the full ansatz for the 2-form can be written as

$$
\mathcal{B}=\frac{1}{2} \mathcal{B}_{M N} \eta^{M} \wedge \eta^{N}+\omega
$$

Thus the construction is specified by constants $\gamma_{N P}^{M}, \beta_{M N P}$ satisfying (4.2), (4.4) and has internal moduli $\mathcal{G}_{M N}, \mathcal{B}_{M N}$ giving $d^{2}$ scalar fields in the compactified theory, in addition to the dilaton, just as for the torus case (the constants $\gamma_{N P}^{M}, \beta_{M N P}$ do not give further moduli). This is sufficient to ensure that the reduced field equations have no dependence on the internal coordinates, but reduction of the action requires that the volume element can also be reduced to give an action that is independent of the internal coordinates, and this requires the further condition

$$
\gamma_{N M}^{N}=0
$$

The construction is very similar to a toroidal compactification, but with the coordinate frame $d y^{M}$ replaced by the $\eta^{M}$ frame, which is 'twisted' by the constants $\gamma_{N P}^{M}$ to give what is sometimes referred to as a 'twisted torus' associated with the $d$-dimensional group $K$. For reduction on an internal space with metric and B-field given by (4.3) and (4.6), Scherk and Schwarz gave an ansatz that allows the truncation of the $n$-dimensional field theory to a $(D=n-d)$-dimensional one that is independent of the internal coordinates $y$. However, for this to be a compactification, one needs an internal space that is compact with the local structure (4.1). If the isometry group $K$ is compact, we can take the internal manifold to be the group manifold $K$ with isometry group $K_{L} \times K_{R}$ with the $\eta^{M}$ the left-invariant Maurer-Cartan one-forms. In general, the internal space must be the quotient $K / \Gamma$ of the group manifold by a discrete subgroup of $\Gamma$ of $K_{L}$, the left action of $K$ on itself [15]. If $K$ 
is non-compact, it is necessary to choose $\Gamma$ so that $K / \Gamma$ is compact in order for the ScherkSchwarz reduction and truncation of a field theory to be extendable to a compactification of string theory. A necessary condition [15] for the existence of such a $\Gamma$ is (4.7). We will assume (4.7) here.

The ansatz for the reduction is the most general one that is invariant under the left action $K_{L}$. For supergravity, the reduction can be viewed as reduction on the group manifold followed by truncation to the $K_{L}$ singlet sector, and the isometry of the internal space $K_{R}$ gives rise to a gauge group $K[15]$. For the full string theory, we wish to consider the full theory without truncation, so we require a compact internal space. For $K$ non-compact, we consider reduction on compact spaces $K / \Gamma$ where $\Gamma$ is a discrete subgroup of $K_{L}$. The left-invariant one-forms $\eta$ on $K$ then give well-defined one-forms on $K / \Gamma$.

Let $x^{\mu}$ be $D$-dimensional space-time coordinates and $y^{M}$ be coordinates on the $d$-dimensional internal space. It is useful to define

$$
\nu^{M}=\eta^{M}(y)+V_{\mu}^{M}(x) d x^{\mu}
$$

where $V^{M}{ }_{\mu}(x)$ are the $d$ Kaluza-Klein vector fields which are gauge fields for the isometry group $K$, with field strength $d V^{M}+\gamma_{N P}^{M} V^{N} \wedge V^{P}$. The full ansatz for the reduction of the metric is [16]

$$
d s^{2}=g_{\mu \nu}(x) d x^{\mu} d x^{\nu}+\mathcal{G}_{M N}(x) \nu^{M} \nu^{N}
$$

where the metric $\mathcal{G}_{M N}(x)$ gives $d(d+1) / 2$ scalar fields. The ansatz for the 2 -form gauge field is

$$
\mathcal{B}=\frac{1}{2} \mathcal{B}_{\mu \nu} d x^{\mu} \wedge d x^{\nu} \wedge+\mathcal{B}_{\mu M} d x^{\mu} \wedge \nu^{M}+\frac{1}{2} \mathcal{B}_{M N} \nu^{M} \wedge \nu^{N}+\omega
$$

with 3 -form field strength $\mathcal{H}=d \mathcal{B}$.

The reduced theory has $2 d$ gauge fields $A_{\mu}^{a}=\left(V^{M}{ }_{\mu}, B_{\mu M}\right)$. The $V^{M}$ are gauge fields corresponding to the isometry group $K_{R}$ of $K$, and in the standard case in which there is no flux $(\beta=0)$ the corresponding generators of gauge transformations $Z_{M}$ satisfy the $K$ commutation relations $\left[Z_{M}, Z_{N}\right]=2 i \gamma_{M N}^{P} Z_{P}$. There are a further $d$ gauge symmetries for which $B_{\mu M}$ are the gauge fields, with generators $X^{M}$. The full reduced theory has a $2 d-$ dimensional gauge group $G$ generated by the $Z_{M}, X^{M}$. The algebra was calculated in [12] and found to be

$$
\begin{aligned}
& {\left[X^{M}, X^{N}\right]=0} \\
& {\left[X^{M}, Z_{N}\right]=2 i \gamma_{N P}^{M} X^{P}} \\
& {\left[Z_{M}, Z_{N}\right]=-3 i \beta_{M N P} X^{P}+2 i \gamma_{M N}^{P} Z_{P}}
\end{aligned}
$$

so that the flux modifies the algebra. The Jacobi identities are satisfied as a result of (4.2) and (4.4). If $K$ is semi-simple and the flux is given by the structure constants, then the term involving the flux $\beta_{M N P}$ can be removed by redefinitions [18], but in general this is not possible [15]. 
Introducing the generators $T_{a}=\left(Z_{M}, X^{M}\right)$ of $G$, the algebra can be written as

$$
\left[T_{a}, T_{b}\right]=i f_{a b}{ }^{c} T_{c}
$$

where

$$
f^{M}{ }_{N P}=f_{N P}^{M}=2 \gamma_{N P}^{M} \quad f_{M N P}=-3 \beta_{M N P}
$$

The gauge group $G$ is a subgroup of the $O(d, d)$ that was a symmetry of the toroidally reduced theory. The reduced action is (2.8).

\section{$5 \quad$ Reductions with Duality Twists}

There is a second type of dimensional reduction which is also often called a Scherk-Schwarz reduction. If a theory in $D+1$ dimensions has a global symmetry $K$, then reducing on a further circle to $D$ dimensions, one can allow a twist around that circle by an element of $K$. We refer to these as reductions with duality twists [2], and will show that while a large class of these are Scherk-Schwarz reductions of the type considered in the previous section, this is not true in general, and the twisted reductions which are not of Scherk-Schwarz type will play an important role. We will be interested here in the case in which a string theory is reduced first on a $d-1$ torus to give a theory in $D+1$ dimensions with global symmetry $O(d-1, d-1 ; \mathbb{Z})$ and reducing on a further circle with an $O(d-1, d-1 ; \mathbb{Z})$ twist. The lowenergy field theory in $D+1$ dimensions in fact has an $O(d-1, d-1)$ continuous symmetry and in the field theory one could twist with an element of $O(d-1, d-1)$, while in the full string theory there is an action of $O(d-1, d-1)$ on the theory, but only the discrete subgroup $O(d-1, d-1 ; \mathbb{Z})$ is a symmetry and the twist must be in this discrete subgroup. This is the typical situation in the examples arising in string theory; there is an action of a continuous group $K$ of which a discrete subgroup $K(\mathbb{Z})$ is a symmetry, and the ansatz uses the action of $K$ but the twist is required to be in $K(\mathbb{Z})[10]$.

Suppose an element $g$ of a continuous group $K$ in $D+1$ dimensions acts on a generic field $\psi$ as $\psi \rightarrow g[\psi]$. For our string theory case, $K=O(d-1, d-1)$. Consider now the twisted dimensional reduction of the theory to $D$ dimensions on a circle of radius $R$ with a periodic coordinate $y \sim y+2 \pi R$. In the twisted reduction, the fields are not independent of the internal coordinate but are chosen to have a specific dependence on the circle coordinate $y$ through the ansatz

$$
\psi\left(x^{\mu}, y\right)=g(y)\left[\psi\left(x^{\mu}\right)\right]
$$

for some $y$-dependent group element $g(y) \in K$. An important restriction on $g(y)$ is that the reduced theory in $D$ dimensions should be independent of $y$. This is achieved by choosing

$$
g(y)=\exp \left(\frac{M y}{2 \pi R}\right)
$$


for some Lie-algebra element $M$. The map $g(y)$ is not periodic around the circle in general, but has a monodromy

$$
\mathcal{M}(g)=\exp M
$$

In the string theory, the monodromy has to be an element of $\mathcal{G}(\mathbb{Z})=O(d-1, d-1)(\mathbb{Z})[10]$.

The reduction for string theory with an $O(d-1, d-1 ; \mathbb{Z})$ twist is straightforward and gives a $D$ dimensional theory with effective action (2.8) and a non-semi-simple gauge group $G$. The coordinates $y^{M}$ split into the coordinates $y^{m}$ on the $(d-1)$-torus with $m=1, . ., d-1$ and the coordinate $y$ on the final circle, $y^{M}=\left(y, y^{m}\right)$, so that the gauge field one-forms are $V^{y}, V^{m}$ and $B_{y}, B_{m}$ with corresponding gauge group generators $Z_{y}, Z_{m}, X^{y}, X^{m}$. It will also be useful to introduce indices $\alpha, \beta=1, \ldots, 2(d-1)$ for the fundamental representation of $O(d-1, d-1)$ so that the gauge generators are $T_{a}=\left(Z_{y}, X^{y}, T_{\alpha}\right)$ where $T_{\alpha}=\left(Z_{m}, X^{m}\right)$. Then the gauge algebra for this reduction is

$$
\left[Z_{y}, T_{\alpha}\right]=M_{\alpha}^{\beta} T_{\beta}
$$

with all other commutators vanishing, and from this the structure constants $f_{a b}^{c}$ can be read off. The scalar potential is [16]

$$
V(\Phi)=e^{a \phi} \operatorname{Tr}\left[M^{2}+M^{t} K M K^{-1}\right]
$$

where $e^{\phi}$ is the modulus corresponding to the radius of the final circle and $a=6 /(D-$ 1) $(D-2)$.

In the basis in which $T_{\alpha}=\left(Z_{m}, X^{m}\right)$, the mass matrix $M_{\alpha}{ }^{\beta}$ has the decomposition

$$
M_{\alpha}{ }^{\beta}=\left(\begin{array}{cc}
w_{m}^{n} & u_{m n} \\
v^{m n} & -\left(w^{t}\right)^{m}{ }_{n}
\end{array}\right)
$$

where $u_{m n}=-u_{n m}$ and $v^{m n}=-v^{n m}$ while $w_{m}^{n}$ is an unconstrained matrix. The algebra (2.7) can then be written as

$$
\begin{aligned}
& {\left[Z_{y}, Z_{m}\right]=w_{m}^{n} Z_{n}+u_{m n} X^{n}} \\
& {\left[Z_{y}, X^{m}\right]=-w_{n}{ }^{m} X_{m}+v^{m n} Z_{n}} \\
& {\left[Z_{n}, Z_{m}\right]=0 \quad\left[X^{m}, X^{n}\right]=0}
\end{aligned}
$$

This is of the same form as (3.4) only if $v^{m n}=0$. Thus the twisted reduction considered here is more general than the Scherk-Schwarz reduction of the type considered in $\$$ whenever $v^{m n}$ is nonzero. Conversely, not all Scherk-Schwarz compactifications can be viewed as dualitytwisted compactifications. (For example, Scherk-Schwarz compactification on a non-abelian semi-simple group manifold $H$ without flux will result in the $Z_{m}$ generating the Lie algebra of $H$ which is not of the form (5.7) in general.) 
Comparing with the general algebra (3.4) in $\$ 3$ we see that in this way we obtain a gauging where $\tilde{\gamma}$ and $\tilde{\beta}$ are zero and all other structure constants are nonzero. The ScherkSchwarz (twisted torus) reductions have non-zero $\gamma=\hat{\gamma}, \beta$, but the reduction with duality twist has a more general algebra in which $\bar{\gamma}$ is also non-zero. We show in the next section how $\tilde{\gamma}$ and $\tilde{\beta}$ can arise in the context of non-geometric shifts and asymmetric orbifolds.

The mass matrix (5.6) is in the Lie algebra of $O(d-1, d-1)$. The subalgebra parameterised by $w_{m}^{n}$ is $G L(d-1, \mathbb{R})$ which acts geometrically on the torus $T^{d-1}$ while $u_{m n}$ parameterises the transformations that shift the $B$-field on the torus by a constant 2 -form. Thus the subalgebra in which $v^{m n}=0$ is the one that can be realised geometrically as diffeomorphisms of $T^{d-1}$ and shifts of the $B$-field and such twists can be realised as standard reductions on a manifold which can be viewed either as a $T^{d-1}$ bundle over a circle with suitable $B$-field ansatz, or as a reduction on a discrete identification of the group manifold for the group $G$ with Lie algebra (5.7). When $v^{m n} \neq 0$, the T-duality twist is non-geometrical and it is this non-geometrical case that is not of the Scherk-Schwarz form of \$4, giving an internal space which is a T-fold [1] rather than a geometrical background.

\section{WZW Models}

We now discuss an example where the full nonabelian structure of the gauge group plays a role. Following the general discussion in $\$ 2$ compactification of the action (2.1) on $\mathbf{T}^{3}$ gives a low energy theory (2.2) with an $O(3,3)$ rigid symmetry and six vector fields in the vector representation $(\mathbf{3}, \mathbf{3})$ of $O(3,3)$. We can choose to gauge an $S O(3) \times S O(3)$ subgroup since the vector representation $(\mathbf{3}, \mathbf{3})$ of $O(3,3)$ decomposes into the adjoint $(\mathbf{3}, \mathbf{1})+(\mathbf{3}, \mathbf{1})$ of $S O(3) \times S O(3) \subset O(3,3)$, to give a gauged theory with action (2.8). There is a natural candidate for the string-theory lifting of this gauging, which is one where the internal CFT is the $S O(3)$ WZW model (or a non-conformal deformation of this that preserves $S O(3) \times S O(3)$ global symmetry) as this would give the same gauge symmetry $S O(3) \times S O(3)$. We will now analyse this case in more detail, showing that the symmetry breaking patterns emerging from the scalar potential are exactly those of the WZW model, supporting our claim that the WZW model is the correct lifting of this gauging. It is remarkable that our potential

(2.14) then provides a complete non-linear form for the potential for the WZW model; we will explore this in more detail elsewhere [29].

From the embedding of $S O(3) \times S O(3) \subset O(3,3)$, the generators $T_{M}$ and $\tilde{T}_{M}$ of the two $S O(3)$ factors $(M=1,2,3)$ are related to the generators $Z_{M}, X^{M}$ by

$$
T=Z+X, \quad \tilde{T}=Z-X
$$


(with indices raised or lowered with $\delta_{M N}$ ). Then the gauge algebra takes the form

$$
\begin{aligned}
& {\left[X^{M}, X^{N}\right]=3 i \epsilon^{M N P} Z_{P}} \\
& {\left[X^{M}, Z_{N}\right]=2 i \epsilon_{N P}^{M} X^{P}} \\
& {\left[Z_{M}, Z_{N}\right]=2 i \epsilon_{M N}^{P} Z_{P}}
\end{aligned}
$$

Comparing with [3.4, we see that this gives $\tilde{\beta}^{M N P}=\epsilon^{M N P}, \gamma_{N P}^{M}=\hat{\gamma}_{N P}^{M}=\epsilon_{N P}^{M}$. Note that gauge algebras with nontrivial $\tilde{\beta}, \hat{\gamma}_{N P}^{M}$ are in this case arising from a geometric reduction on the $S O(3)$ group manifold (or $S^{3}$ ) with flux.

The scalar fields in the $O(3,3) / O(3) \times O(3)$ coset space can be parameterised by a matrix $\varphi^{\alpha \alpha^{\prime}}$ where $\alpha=1,2,3$ takes values in the adjoint representation of one $S O(3)$ and $\alpha^{\prime}=1,2,3$ takes values in the adjoint representation of the other $S O(3)^{\prime}$. It is easy to see that the potential (2.14) has a leading term which is cubic in $\varphi^{\alpha \alpha^{\prime}}$ and takes the form

$$
W(\mathcal{K})=-4+\epsilon_{\alpha \beta \gamma} \epsilon_{\alpha^{\prime} \beta^{\prime} \gamma^{\prime}} \varphi^{\alpha \alpha^{\prime}} \varphi^{\beta \beta^{\prime}} \varphi^{\gamma \gamma^{\prime}}+O\left(\varphi^{4}\right)=-4+\operatorname{det}(\varphi)+O\left(\varphi^{4}\right)
$$

The full potential is non-polynomial in $\varphi^{\alpha \alpha^{\prime}}$. In this leading-order approximation, $\varphi$ can be thought of as Higgs field that transforms in the bi-adjoint representation of the gauge symmetry and thus to start with there are nine scalar fields (plus a dilaton). The full symmetry is restored at $\varphi=0$. The potential allows for a single flat direction and the moduli fields can acquire a nonzero expectation value $\varphi^{\alpha \alpha^{\prime}}=\delta^{\alpha 3} \delta^{\alpha^{\prime} 3} v$. When the vacuum expectation value $v$ is nonzero, then the symmetry is broken to $S O(2) \times S O(2)$. By expanding around this vev, it is easy to see that out of the nine scalar fields, one remains massless for all values of $v$ corresponding to the flat direction. We will show in [29] that this is an exact flat direction of the full non-polynomial potential. There are four would-be goldstone bosons that are eaten by the four gauge fields which then acquire a mass of order $v$. Of the remaining four, two become massive and two become tachyonic.

This symmetry breaking pattern is exactly what one would obtain by compactifying the bosonic string on a three-dimensional $S O(3)$ group manifold with three-form flux given by the invariant 3-form so that it corresponds to a WZW model. Compactifying on the $S O(3)$ $W Z W$ model one would obtain $S O(3)_{L} \times S O(3)_{R}$ as the spacetime gauge symmetry. If $J^{a}$ and $\tilde{J}^{a}$ are the currents of $S O(3)_{L}$ and $S O(3)_{R}$ respectively then there is an exactly marginal operator in the worldsheet conformal field theory $\int a J^{3} \tilde{J}^{3}$ where $a$ is a real coefficient. As a result, there is precisely one flat direction (up to gauge equivalence). The moduli space of this compactification is one-dimensional, labelled by this real number $a$ which we can identify with $v$ above. Moreover, for nonzero $a$, the addition of this perturbation breaks the symmetry to $S O(2)_{L} \times S O(2)_{R}$ generated by $J_{3}$ and $\tilde{J}_{3}$.

We are interested here in the large $k$ limit of the WZW model that is nearly classical. However, the symmetry-breaking pattern does not depend on $k$, so a quick way to verify this pattern is to look at the WZW model with $k=1$ described by a single boson $X$ near the 
self-dual radius. If we compactify the bosonic string on a circle at the self-dual radius, there is one flat direction for the spacetime potential corresponding to changing the radius. Giving a nonzero vev to the modulus field corresponds to moving slightly away from the self-dual radius which breaks the symmetry to $S O(2)_{L} \times S O(2)_{R}$ generated by the worldsheet currents $\partial X$ and $\tilde{\partial} X$. It is easy to see the spectrum of states is exactly the same as above.

It is thus nontrivial that for the natural generalisation of the Scherk-Schwarz potential that we have proposed, the quadratic term is absent and the potential correctly captures the symmetry breaking pattern expected from string theory. Note also that the vacuum energy $g^{2} W$ at the minimum of the potential does not vanish but instead has a small negative value that goes as $-4 g^{2}$. This acts as a tadpole for the dilaton and the equations of motion of the dilaton would not be satisfied unless we adjust the total central charge to be 26 . The gauge coupling constant $g^{2}$ corresponds to $1 / k$ and hence this correction to the central charge goes as $-1 / k$. Recall that the central charge of the $S O(3)$ model is $\frac{3 k}{k+2}$ which for large $k$ goes as $3-\frac{6}{k}+\ldots$. The correction $-\frac{6}{k}$ is the leading $\alpha^{\prime}$ correction. It is interesting that our generalized potential is able to capture this subleading correction even though we are working in the framework of supergravity.

In superstring theory, we compactify the theory on a super WZW model at level $k$ which factorizes in terms of three Majorana-Weyl fermions and a bosonic WZW model at level $k-2$. Hence, our analysis above applies without much change. Note that now the corrections to the central charge is exactly $-\frac{6}{k}$ and there are no further $\alpha^{\prime}$ corrections.

To obtain a supersymmetric, tachyon-free compactification of the Type-II string one can consider, for example, $\mathbf{A d S}_{\mathbf{3}} \times \mathbf{S}^{\mathbf{3}} \times \mathbf{K} \mathbf{3}$ that arises as a near-horizon limit of the NS5-F1-P black hole. The $\mathbf{S}^{\mathbf{3}}$ factor is then described by a super WZW model. The bulk supergravity has a dual description in terms of a boundary sigma model with $(4,4)$ superconformal symmetry. In the boundary theory, the $S U(2) \times S U(2)$ symmetry of the bulk appears as the R-symmetry. By turning on various operators in the boundary CFT that break the $(4,4)$ superconformal symmetry to $(2,2)$ superconformal symmetry one can reduce the R-symmetry from $S U(2) \times S U(2)$ to $U(1) \times U(1)$. These will correspond to interesting renormalisation group flows in the boundary CFT. In the bulk supergravity, the description of this symmetry breaking pattern far away from the symmetric point would require the full nonlinear structure of the effective potential above that encapsulates the higher order interactions.

\section{T-Folds and Asymmetric Orbifolds}

We have seen that a particular class of gaugings (those with gauge algebra (4.13)) arise from Scherk-Schwarz reductions with flux, while another class (with gauge algebra (5.4)) arises from reductions with duality twists, but these constitute only a restricted class of possible 
gaugings. For the remainder of this paper, we wish to investigate the question of which other gaugings can have a string theory origin, and we will consider in particular stringy generalizations of the Scherk-Schwarz reduction and orbifold constructions, which are closely related. Many of our considerations will apply equally to the bosonic, heterotic and type II strings. Our initial goal will be to explore the classical structure of such a generalization using symmetry considerations. We will see that our proposed generalization encompasses the duality-twisted reductions along with Scherk-Schwarz reductions and thus includes nongeometric possibilities that are classically consistent. We postpone questions of quantum consistency such as modular invariance for future investigation.

The starting point is then a choice of gauge group $G$ which is a $2 d$ dimensional subgroup of $O(d, d)$ with structure constants $f_{a b}{ }^{c}$ such that the fundamental representation of $O(d, d)$ becomes the adjoint of $G$ under the embedding of $G$ in $O(d, d)$. Then the truncation to the common sector of the bosonic action in $D$ dimensions is given by (2.8) with the definitions (2.9), (2.10), (2.12).

By construction, the potential of gauged supergravity (2.14) proposed in \$2 gives the Scherk-Schwarz potential when the structure constants take the form (3.2). It is also easy to see that when the structure constants take the form (5.4), the potential reduces to the potential (5.5) of the duality-twisted compactifications. Note that the duality twists with nonzero $v^{m n}$ in (5.7) are more general than the Scherk Schwarz type because the T-duality group $O(d, d, \mathbb{Z})$ contains additional information about winding modes that goes beyond supergravity. For example, as shown in [2], when the duality twist is a general element of $O(d-1, d-1, \mathbb{Z})$ that is not an element of the geometric of $S L(d-1, \mathbb{Z})$ of the torus, the theory at the minimum of the potential is described by an asymmetric orbifold. Such a stringy twist clearly goes beyond the usual geometry underlying Scherk-Schwarz reductions.

\section{$7.1 \quad$ T-dual Shifts}

We now show that the generalization (3.4) for the gauge algebra proposed in $\$ 3$ is realized naturally in the context of general orbifolds. We have seen that one can generate a class of non-geometric twists from geometric ones by acting with $O(d-1, d-1, \mathbb{Z})$ T-duality transformations. To go further and generate more general gauge algebras it is natural to consider acting with $O(d, d, \mathbb{Z})$ T-duality transformations. In particular, it is natural to consider T-dualising the circle with coordinate $y$ that carries the monodromy. However, there is an obvious and immediate obstruction to this: translation in the circle direction is not an isometry as the ansatz (5.1) introduces explicit $y$-dependence into the background so that the moduli of the internal torus are $y$-dependent. T-duality as normally formulated requires that translation in the direction in which one dualises to be an isometry. However, as we shall review below, for elliptic twists there is a point in the moduli space at which 
$y$-translations become an isometry [2] and in this case one can T-dualise. Moreover, we showed in 2] that at such special points in moduli space, the twisted reduction becomes equivalent to an orbifold by a $\mathbb{Z}_{n}$ symmetry of the torus CFT together with an order $n$ shift along a spectator circle [2]. As we shall see, the T-dual is again an orbifold, but now by a symmetry involving a shift in the T-dual circle. In both cases we can consider the effective compactified supergravity theory and the special points in moduli space correspond to minima of the scalar potential. In the case considered in 2] with shifts in the original circle, the potential gives information about deformations away from the special point in moduli space, and it is natural to conjecture that this applies for the new orbifold with a T-dual shift as well. This would be remarkable, as it is unclear how to formulate string theory in such circumstances - the background would be non-geometric and of a kind more general than the T-folds proposed in [1]. We now proceed to discuss this construction in more detail.

The relation between duality-twisted reductions and orbifolds was explored in [2]. The duality twist is said to be of the 'elliptic' type if the matrix $M$ in (5.2) is a compact generator of $O(d-1, d-1)$ and is thus conjugate to an element of the compact subgroup $O(d-1) \times$ $O(d-1)$. One can show that for an elliptic twist the monodromy $\mathcal{M}$ is always of finite order, say $n$, so that $\mathcal{M}^{n}=1$. This is basically because a discrete rotation is of finite order. It was shown in 2] that for duality twists which are of the elliptic type, the theory at the minimum of the potential is given by an orbifold. The monodromy matrix $\mathcal{M}$ generates a $\mathbb{Z}_{n}$ subgroup of the discrete duality group. Generically, the duality group maps one point of the moduli space to another point. However, the minimum of the potential occurs at a value of the moduli which is left fixed by this $\mathbb{Z}_{n}$ group. At this point, then, the $\mathbb{Z}_{n}$ that leaves it invariant is a proper symmetry of the theory at that point. One can therefore construct an orbifold utilizing this $\mathbb{Z}_{n}$ symmetry. The orbifold action in this case works as follows. Consider an $n$-fold cover of the circle and let the coordinate on this larger circle be $Y$ (so that effectively $Y=n y$ ) with periodicity $2 \pi R_{0}$ with $R_{0}=n R$. Now, consider an orbifold action that combines the order $n$ twist generated by $\mathcal{M}$ with an order $n$ shift along this larger circle, i.e. a shift by

$$
Y \rightarrow Y+2 \pi R_{0} / n
$$

In the the theory over the larger circle, all fields are periodic going around the coordinate $Y$. The orbifolding results in a nontrivial bundle going around the original circle coordinate $y$ with monodromy $\mathcal{M}$.

One obvious way to generalise this structure is then to T-dualise along the circle direction. T-duality takes the circle with radius $R_{0}$ and coordinate $Y$ to a dual circle with radius $\tilde{R}_{0}=1 / R_{0}$ and coordinate $\tilde{Y} \sim \tilde{Y}+2 \pi \tilde{R}_{0}$. The $\mathbb{Z}_{n}$ orbifold in the dual description is then generated by an element $\alpha$ corresponding to the same duality twist as before but now 
accompanied by an order $n$ shift in the T-dual circle coordinate $\tilde{Y}$

$$
\tilde{Y} \rightarrow \tilde{Y}+2 \pi \tilde{R}_{0} / n
$$

Given the T-duality symmetry of the underlying string theory, one can always carry out the T-duality along the $Y$ circle in the orbifold conformal field theory. Moreover, modular invariance of the original orbifold ensure the modular invariance of the T-dual orbifold.

To understand the meaning of the shift along $\tilde{Y}$ more concretely, recall that after compactifying along the circle in the $Y$ direction, the momenta and winding along this circle take values in a lattice $\Gamma^{1,1}$. A basis for the string Hilbert space includes the states $|m, w\rangle$ that carry $m$ units of quantized momentum and wrap $w$ times along $Y$. Now, an order $n$ shift along $Y$ acts on these states as

$$
|m, w\rangle \rightarrow \exp (2 \pi i m / n)|m, w\rangle
$$

Under T-duality, the quantum numbers $(m, w)$ are related to the corresponding $(\tilde{m}, \tilde{w})$ quantum numbers in the dual theory by $m=\tilde{w}$ and $w=\tilde{m}$. Hence, an order $n$ shift along the T-dual $\tilde{Y}$ coordinate acts on these states as

$$
|\tilde{m}, \tilde{w}\rangle \rightarrow \exp (2 \pi i \tilde{w} / n)|\tilde{m}, \tilde{w}\rangle .
$$

This defines the $\tilde{Y}$ shift in the CFT.

A simple nontrivial supersymmetric example of such an orbifold is the following. Consider Type-II string theory on $\mathbf{T}^{\mathbf{4}} \times \mathbf{S}^{\mathbf{1}}$. One can consider a $\mathbb{Z}_{2}$ orbifold action that acts as an inversion on all coordinates of the $\mathbf{T}^{4}$ accompanied by an order two shift along the circle. Without the shift, one would obtain Type-II string on (the $\mathbf{T}^{\mathbf{4}} / \mathbb{Z}_{\mathbf{2}} \times \mathbf{S}^{\mathbf{1}}$ orbifold limit of) $\mathbf{K} \mathbf{3} \times \mathbf{S}^{\mathbf{1}}$. However, with the shift, the twisted states are massive and one will get a theory with the same number of supersymmetries as the $\mathbf{K} \mathbf{3} \times \mathbf{S}^{\mathbf{1}}$ compactification but different massless spectrum. Note that the order two shift could be either be of type (7.3) or (7.4) with $n=2$, depending on whether one is shifting along the $Y$ circle or along the the dual $\tilde{Y}$ circle. In both cases, the resulting orbifold is modular invariant and quantum consistent.

Note, however that the orbifold CFT exists only at the minimum of the potential and not away from it. At the minimum of the potential, the moduli fields are at the fixed point of the discrete symmetry. As a result, one can show that with the ansatz (5.1), the moduli fields are independent of the coordinate $Y$ or $y$. One way to understand this fact is to note that the Scherk-Schwarz potential arises essentially from the gradient energy of the fields with the ansatz (5.1). At the minimum, there is no gradient energy, which means that the fields are independent of $y$. In this case we have then translation invariance along $y$, which allows us to apply the usual T-duality rules.

When we move away from the minimum of the potential, the moduli of the theory are no longer at the fixed point of the discrete symmetry generated by $\mathcal{M}$. In this case, there 
is a nontrivial dependence on the coordinate $y$ for the fields given by the ansatz (5.1). Now, without translation invariance along $y$, we can no longer use the naive T-duality rules. Thus, even though the theories at the minimum are related to each other by T-duality one would expect that the theories away from the minimum are not directly related by a simple T-duality but in a certain sense exchange the dependence on momentum modes with the dependence on winding modes.

\subsection{Asymmetric Twists and Shifts}

The next level of generalization would be to allow for a shift in both $Y, \tilde{Y}$ directions and in this way obtain a gauge algebra in (3.4) where all $\gamma, \beta, \tilde{\gamma}$, and $\tilde{\beta}$ are non-vanishing. The gauge algebra in this case would be

$$
\left[Z_{y}, T_{\alpha}\right]=M_{\alpha}^{\beta} T_{\beta}
$$

and

$$
\left[X^{y}, T_{\alpha}\right]=\tilde{M}_{\alpha}^{\beta} T_{\beta}
$$

with

$$
[M, \tilde{M}]=0
$$

If $M$ and $\tilde{M}$ are distinct but mutually commuting and generate subgroups of order $n$ and $m$ respectively then the theory at the minimum would be described by an $\mathbb{Z}_{n} \times \mathbb{Z}_{m}$ orbifold. The orbifold action is generated by an element $\alpha$ corresponding to an $M$ twist accompanied by an order $n$ shift along the $y$ circle and $\beta$ corresponding to an $\tilde{M}$ shift accompanied by a order $m$ shift along $\tilde{Y}$. Note that even though that the theory at the minimum of the potential will be described by a regular asymmetric orbifold, in the theory away from the minimum, the fields would have unusual non-geometric dependence on both winding and momentum modes.

Asymmetric orbifolds are severely constrained by modular invariance and generically an asymmetric twist and a shift would not lead to a modular invariant theory [20]. Our analysis here is entirely classical and the quantum consistency of the theory is not guaranteed at this level, so that quantum consistency will lead to extra constraints. However, any background that is T-dual to a consistent background will be consistent. Moreover, there do exist several nontrivial examples of orbifolds with asymmetric twists and shifts that are quantum consistent and which cannot be obtained as a T-dual of a symmetric orbifold. See, for example, 21]. Thus, the general algebra (3.4) that we have used as the basis of our formalism of gauged supergravity can certainly be realized within string theory at the minimum of the potential. 


\section{Generalised T-duality and New Non-Geometric Con- structions}

For reductions with duality twists, conventional T-duality cannot be applied to the circle on which the theory is twisted. In this section, we generalise the standard T-duality to obtain T-duals of configurations that have no conventional T-dual, obtaining new classes of non-geometric backgrounds. We will argue that these must be good string backgrounds. In this section, we will use the picture [1] in which taking a T-dual corresponds to change of choice of polarisation in a doubled formalism. We will then show in $\$ 9$ how this emerges naturally from string field theory.

Consider a reduction with duality twist, with a reduction on $T^{d-1}$ followed by reduction on a circle with an $O(d-1, d-1 ; \mathbb{Z})$ T-duality twist. The dependence on the coordinate $y$ of the final circle is through the $O(d-1, d-1)$ group element

$$
g(y)=\exp \left(\frac{M y}{2 \pi R}\right)
$$

with monodromy

$$
\mathcal{M}(g)=\exp M
$$

in $O(d-1, d-1 ; \mathbb{Z})$. Taking the T-dual on one of the circles in $T^{d-1}$ is straightforward as there is no dependence on the coordinates of $T^{d-1}$. For example, for geometric twists with monodromy in $S L(d-1, \mathbb{Z})$, the reduction is equivalent to reduction on a $d$-dimensional compact space which is a $T^{d-1}$ bundle over $S^{1}$ [10]. The translations in the fibre directions are isometries and so T-duality along a fibre direction can be done according to the standard Buscher rules. In some cases this gives another geometric reduction, while in others it gives a T-fold [1]. However, T-duality along the $y$-direction is problematic, as the background depends explicitly on $y$. In the geometric reduction, the torus bundle has no isometry in the $y$ direction so that the Buscher rules cannot be applied.

However, there is evidence that it should be possible to take a T-dual in the $y$ direction. Firstly, the circle has both momentum and winding degrees of freedom, although the momentum $p_{y}$ is not constant along the circle and depends on $y$. Nonetheless one might expect an equivalent formulation with momentum and winding interchanged, now with the 'winding charge' depending on the circle coordinate. As we shall see, a natural way this might be achieved is by a simple rewriting with $y$ exchanged with its dual coordinate $\tilde{y}$.

Secondly, for elliptic twists, there are points in moduli space in which the reduction becomes independent of $y$, and becomes equivalent to an orbifold with a shift in the $y$ direction. This can then be dualised to give an orbifold with a dual shift in the $\tilde{y}$ direction. Before dualising, we know that there is no obstruction to moving away from the special orbifold point in moduli space, but there is an energy cost, as the moduli become scalar fields 
of the dimensionally reduced theory, and moving way from the orbifold point involves moving away from the minimum of the scalar potential. However, the T-dual orbifold is the same conformal field theory, but written in different variables. This means that it must be possible to move away from the orbifold point in moduli space in the T-dual theory also. Moreover, this deformation is readily understood in the dimensionally reduced effective field theory. We have reviewed how the T-duality acts on the effective field theory in $D$ dimensions, giving a new effective potential. However, the T-duality can be viewed as a change of variables to a T-dual set of fields, so that the new potential can be thought of as being the old one written in the new variables. Moving away from the minimum of the potential is clearly possible in the reduced theory, again at the cost of increase in potential energy.

Our potential (5.5) allows us to go away from the minimum in the low-energy effective theory and gives a way of thinking about the off-shell duality. The key question is whether one can lift this to the full theory, allowing a deformation to a new kind of non-geometric background. One would expect that the T-dual of an orbifold with $Y$-shift (recall that $Y=n y$ is the coordinate on the $n$-fold cover of the circle), which is an orbifold with a shift along $\tilde{Y}$, is a minimum of a potential which is obtained by gauging the algebra that is $\mathrm{T}$ dual to the algebra that was gauged to obtain (5.5). Under T-duality in the $y$ direction, the element $Z_{y}$ is conjugated to the element $X_{y}, X_{y}=T Z_{y} T^{-1}$. Thus, in the T-dual description when we are shifting along $\tilde{Y}$ direction, the gauge algebra for this reduction is expected to be

$$
\left[X^{y}, T_{\alpha}\right]=\tilde{M}_{\alpha}{ }^{\beta} T_{\beta}
$$

with all other commutators vanishing. This is obtained simply by acting on the algebra (5.4) by the T-duality transformation along the $y$ direction. In this case, starting with a situation with non-vanishing $\gamma$ and $\beta$ but vanishing $\tilde{\gamma}$ and $\tilde{\beta}$ in our general gauge algebra (3.4), one would obtain a gauge algebra with non-vanishing $\tilde{\gamma}$ and $\tilde{\beta}$ but vanishing $\gamma$ and $\beta$.

If moving away from the special point in moduli space for the orbifold with $y$-shift gives a twisted reduction with a twist in the $y$ direction, the natural guess is that doing the same for the orbifold with dual $\tilde{y}$-shift should give a twisted reduction with a twist in the $\tilde{y}$ direction. That is, in reducing on the circle, dependence on the dual coordinate $\tilde{y}$ is introduced through the twist

$$
g(\tilde{y})=\exp \left(\frac{M \tilde{y}}{2 \pi \tilde{R}}\right)
$$

so that the reduction is of the same form as before, but with $y$ and $\tilde{y}$ interchanged. This appears rather trivial from the conformal field theory viewpoint, but the change to a background with dependence on the dual coordinate takes us away from the realm in which there is a local spacetime description.

It is useful to consider these constructions from the point of view of conformal field theory. A state in the conformal field theory on the circle is characterised by momentum $n / R$ and 
winding number $w$ for integers $n, w$ and is represented by the tensor product of a state

$$
|n, w\rangle
$$

with a state in the oscillator Fock space. One can instead perform a Fourier transform and represent a state in a position basis with two periodic coordinates $y, \tilde{y}$ of periods $2 \pi R$ and $2 \pi \tilde{R}=2 \pi / R$ respectively

$$
|y, \tilde{y}\rangle=\sum_{n, w} e^{i n y / R} e^{i w \tilde{y} / \tilde{R}}|n, w\rangle
$$

T-duality interchanges $n$ with $w$ or $y$ with $\tilde{y}$. In the conformal field theory, $n$ and $w$ or $y$ and $\tilde{y}$ are on an equal footing, and can be viewed as living on the doubled space, the 2-torus with coordinates $y$ and $\tilde{y}$. To make contact with the conventional spacetime picture, one must choose a polarisation; one picks one of the two coordinates to be the spacetime coordinate appearing in the sigma-model action, and the other to be the dual coordinate [1. T-duality acts to change the polarisation from the one in which $y$ is a spacetime coordinate to the one in which $\tilde{y}$ is. The statement that T-duality is a symmetry implies that the physics is independent of the choice of polarisation and that both give the same physical results.

The conformal field theory on the torus $T^{d-1}$ is completely specified by a choice of modulus taking values in the coset space $O(d-1, d-1) / O(d-1) \times O(d-1)$ identified under the T-duality group, and can be represented locally by a choice of metric $g$ and $B$-field on the torus $T^{d-1}$, which can be combined into $E=g+B$. There is a natural action of $O(d-1, d-1)$ on the moduli space (acting on $E$ through fractional linear transformations). In the twisted reduction, the moduli depend on the coordinate $y$ through the $O(d-1, d-1)$ transformation (8.1), giving a bundle of conformal field theories over $S^{1}$ with moduli $E(y)$. This is also a bundle over the doubled torus in which there is no dependence on the dual coordinate $\tilde{y}$, so that the bundle over the dual circle is trivial. Now, in this doubled picture one can simply choose the other polarisation, so that $\tilde{y}$ is now viewed as the spacetime coordinate, and $y$ is the dual coordinate. In this polarisation, there is no twisting on the spacetime circle, but there is dependence on the dual coordinate through (8.1). This is of course non-geometric, and cannot be understood in the spacetime alone. On relabelling $y \leftrightarrow \tilde{y}$, one obtains the T-dual picture in which $y$ is the spacetime coordinate but there is a twist on the dual circle through (8.4). From the point of view of conformal field theory, it is just as natural to introduce a $\tilde{y}$-twist as it is to introduce a $y$ twist.

The general twisted construction is to introduce both a $y$-twist and a $\tilde{y}$ twist, so that the dependence of the moduli $E(y, \tilde{y})$ on $y$ and $\tilde{y}$ is through an $O(d-1, d-1)$ transformation

$$
g(y, \tilde{y})=\exp \left(\frac{M y}{2 \pi R}\right) \exp \left(\frac{\tilde{M} \tilde{y}}{2 \pi \tilde{R}}\right)
$$

where the mass-matrices $M, \tilde{M}$ are required to commute and the monodromies

$$
\mathcal{M}(g)=\exp M \quad \tilde{\mathcal{M}}(g)=\exp \tilde{M}
$$


are both in $O(d-1, d-1, \mathbb{Z})$. If $g$ depends just on $\tilde{y}$, it is T-dual to a $\mathrm{T}$-fold construction in which the twist depends only on $y$, so that it is locally geometric, consisting of local spacetime patches glued together using T-dualities, gauge transformations and diffeomorphisms. However, the general case in which it depends on both $y$ and $\tilde{y}$ will not be T-dual to anything that is even locally geometric, as there is dependence on both $y$ and $\tilde{y}$.

In special cases in which the twists are elliptic, there will be fixed points in moduli space at which dependence on $y, \tilde{y}$ drops out, giving an orbifold with shifts in both $y$ and $\tilde{y}$. The generalised twisting over the $\tilde{y}$ circle thus provides a way of moving away from the orbifold point in moduli space.

There is also the possibility of intermediate cases, with special points in moduli space in which the dependence on $\tilde{y}$, say, drops out but the dependence on $y$ does not, so that the result can be thought of as a twisted reduction with twist (8.1) orbifolded by the action of $\tilde{\mathcal{M}}$ together with a shift in the $\tilde{y}$ direction. For example, with $d=3, O(d-1, d-1)$ is locally $S L(2) \times S L(2)$, and if the $\tilde{y}$ twist is an elliptic element of one $S L(2)$ and the $y$ twist is say a parabolic twist in the other $S L(2)$, there will be fixed points in the moduli space at which the $\tilde{y}$ dependence drops out in this way, but there will be no points at which the $y$-dependence drops out.

\section{$9 \quad$ String Field Theory and Doubled Geometry}

\subsection{String Field Theory}

A proper framework for thinking about this generalized T-duality is string field theory, which was developed for toroidal compactification in 22]. One can expand a string field $|\Psi\rangle$ in a basis given by the first quantized string theory defined by a conformal field theory. If the conformal field theory has a circle factor then a state in the first-quantized Hilbert space is labelled as $|m, w, x, I\rangle$ where $m$ and $w$ are the momentum and winding along the circle as above, $x^{\mu}$ are coordinates for the remaining dimensions and $I$ denotes generically all other indices labeling the state. The string field would then have an expansion $|\Psi\rangle^{R}=\sum \Psi_{m, w, I}^{R}(x)|m, n, x I\rangle^{R}$ where the superscript denotes that the string field is defined at radius $R$ of the circle (and the summation includes an integration over $x$ ). Each component $\Psi_{m, w, I}(x)$ in this expansion is a spacetime field. Now, clearly one expects that

the string field $|\Psi\rangle^{R}$ defined at a radius $R$ is T-dual to the string field $|\Psi\rangle^{\tilde{R}}$ defined at the T-dual radius $\tilde{R}=1 / R$ with Buscher-like T-duality rules that will relate the fields $\Psi_{m, w, I}^{R}(x)$ to the fields $\Psi_{\tilde{m}, \tilde{w}, I}^{\tilde{R}}(x)$. Note that the Buscher rules apply to only the lowest components of this tower of states $\Psi_{0,0, I}$ but clearly a generalization does exist since T-duality is an exact map that relates the Hilbert spaces of the first quantized string theories at radius $R$ and $\tilde{R}$. Given the rules of how the basis vectors $|m, n, I\rangle^{R}$ are related to $|\tilde{m}, \tilde{w}, I\rangle^{\tilde{R}}$, which follow 
from the T-duality map of the circle conformal field theory, one knows how the fields $\Psi_{m, w, I}^{R}$ are related to $\Psi_{\tilde{m}, \tilde{w}, I}^{\tilde{R}}$. These are the rules that must be used in doing the T-duality along the circle $y$ in the situation at hand.

Instead of using the basis of states $|m, w, x, I\rangle$, we can Fourier transform to a positionbasis of states $|y, \tilde{y}, x, I\rangle$ depending on the periodic coordinates $y, \tilde{y}$. More generally, for configurations with a $T^{d}$ fibration, the position basis will depend on positions $x^{\mu}$ in the non-toroidal directions, coordinates $y^{i}(i=1, \ldots, d)$ on $T^{d}$ and dual coordinates $\tilde{y}_{i}$, providing a basis of states $\left|x^{\mu}, y^{i}, \tilde{y}_{i}, I\right\rangle$, and expanding a string field will then give an infinite series of fields $\phi_{I}\left(x^{\mu}, y^{i}, \tilde{y}_{i}\right)$ (with a field corresponding to each element in a basis of the oscillator Fock space) depending on the dual coordinates $\tilde{y}$, as well as $x, y$. The $x, y, \tilde{y}$ are coordinates for the 'doubled space' used in [1], and the infinite set of fields are then fields $\phi\left(x^{\mu}, y^{i}, \tilde{y}_{i}\right)$ on this doubled space. T-duality transformations can then take a conventional field depending only on $x, y$ to one depending on $x, \tilde{y}$, or to ones depending on $x, y, \tilde{y}$, and the general solutions of the string field theory equations will give fields depending on $\tilde{y}$, as well as $x, y$.

\subsection{Doubled Geometry}

String field theory then leads us to consider fields on the doubled geometry with coordinates $x^{\mu}, y^{i}, \tilde{y}_{i}$, and in [1] it was shown how to formulate string theory as a constrained sigma-model with target space given by this doubled geometry. In this section, we discuss this geometry for the case of reductions with duality twists and their T-duals. Reduction on $T^{d}$ followed by a reduction on $S^{1}$ with a geometric twist with monodromy in the group $S L(d, \mathbb{Z})$ of large diffeomorphisms of $T^{d}$ can be understood as a compactification on a $T^{d}$ bundle over $S^{1}$ [10]. Reduction on $T^{d}$ followed by a reduction on $S^{1}$ with a non-geometric twist with monodromy in the T-duality group $O(d, d ; \mathbb{Z})$ on $T^{d}$ gives a T-fold (which of course is not a manifold in general) but the doubled geometry is a bundle with fibres given by the doubled torus $\bar{T}^{2 d}$ with coordinates $y, \tilde{y}$. This is a manifold, as $O(d, d ; \mathbb{Z}) \subset S L(2 d ; \mathbb{Z})$ acts geometrically on the doubled torus. The base space is a circle with coordinate $u$, say, and strings on this circle can be formulated in terms of a doubled circle $\bar{T}^{2}$ with coordinates $u, \tilde{u}$. A generalised T-duality on the $u$-circle takes a $\bar{T}^{2 d}$ bundle over the $u$-circle to a $\bar{T}^{2 d}$ bundle over the dual $\tilde{u}$-circle, corresponding to a reduction with twist over the $\tilde{u}$-circle, and the general nongeometric reduction of this type will correspond to a doubled geometry which is a bundle with doubled fibres $\bar{T}^{2 d}$ with coordinates $y, \tilde{y}$ and a doubled base $\bar{T}^{2}$ with coordinates $u, \tilde{u}$, corresponding to a reduction with twists on both the $u$-circle and the dual $\tilde{u}$-circle. This doubled construction is closely related to what has become known as 'generalised geometry' (see e.g. [30]), and in fact can be thought of as a further generalisation of this [1]. This geometry will be explored further elsewhere. 


\section{Discussion}

We have seen that general non-geometric backgrounds have dependence both on the conventional coordinates $y$ and dual coordinates $\tilde{y}$, and that fields in general depend on both $y$ and $\tilde{y}$. The extension of T-duality to circles for which the natural $U(1)$ action is not isometric necessarily leads to such backgrounds. In general, one would expect momentum modes to be sensitive to the $y$-dependence of a background, while momentum modes would be sensitive to the $\tilde{y}$-dependence, so that different probes would 'see' different backgrounds. A world-sheet approach to extending T-duality to backgrounds without isometries was proposed in [23], and it would be interesting to compare this with our construction.

An important class of backgrounds arise from reductions with duality twists, with monodromy $\mathrm{M}$ around a circle with coordinate $y$, and we have considered the example in which this is a T-fold corresponding to a bundle whose fibres are CFT's on $T^{n}$, with moduli depending on $y$. A T-duality $T$ on the $T^{n}$ fibres takes this to a twisted reduction with conjugate monodromy $T \mathcal{M} T^{-1}$. We propose that a generalised T-duality on the base circle of the original background with coordinate $y$ takes this to a twisted reduction over the dual circle, with moduli now depending on the dual coordinate $\tilde{y}$, with monodromy $\mathrm{M}$ on the $\tilde{y}$ circle. In the example of a $T^{3}$ with constant flux, one T-duality takes this to a $T^{2}$ bundle over $S^{1}$ with parabolic twist, a further T-duality in a fibre direction takes this to a T-fold with monodromy $\mathcal{M} \in O(2,2 ; \mathbb{Z})$ and finally a third T-duality on the base circle takes this to a bundle of $T^{2}$ CFT's over the dual circle with the same monodromy $\mathcal{M} \in O(2,2 ; \mathbb{Z})$.

An interesting example of geometries with dependence on a dual coordinate has already appeared in the literature [24, 25, 26, 27]. A Kaluza-Klein monopole solution of string theory (given by the product of Euclidean Taub-NUT with 6-dimensional Minkowski space) is T-dual to a NS 5-brane, smeared over one of the four transverse directions, which is compactified to a circle [28]. A localised version of the NS 5-brane compactified on a circle is obtained by taking an infinite periodic array of parallel NS 5-branes along a line, and then periodically identifying that line. This now has non-trivial dependence on the coordinate $y$ of that circle, but is independent of the dual coordinate $\tilde{y}$. On T-dualising, one would expect $\tilde{y}$ to become the coordinate $x$ on the $S^{1}$ fibre of the Kaluza-Klein monopole, and $y$ to become the dual coordinate $\tilde{x}$. In [24, 25, 26], evidence was given that the T-dual of the localised NS 5 -brane should be a version of the Kaluza-Klein monopole with non-trivial dependence on the dual coordinate $\tilde{x}$, arising from the dependence of the NS 5-brane on $y$, so that whereas momentum modes experience a throat-like geometry in the 5-brane, string winding modes feel a stringy throat-like structure of the Kaluza-Klein monopole. Non-trivial $\tilde{y}$ dependence of the Kaluza-Klein monopole can be understood as arising from world-sheet instantons [24], and this has been verified by linear sigma-model calculations [25, 26. It was also argued in 24] that this T-duality might be understood in terms of a T-duality of higher modes of the string field. 
Conventional effective supergravity theory in 10-dimensions is clearly inadequate for the study of general non-geometric string backgrounds, although the effective supergravity theory arising from reduction on a non-geometric background does provide useful information. There is clearly much that needs to be done to obtain a better understanding of the doubled geometry arising in this context, and the generalisations involving U-duality and brane wrapping modes.

\section{Acknowledgements}

We would like to thank ICTP and the Institute for Mathematical Sciences, Imperial College for hospitality and support. This work was partially supported by an EPSRC visiting fellowship.

\section{References}

[1] C. M. Hull, JHEP 0510 (2005) 065, arXiv:hep-th/0406102.

[2] A. Dabholkar and C. Hull, JHEP 0309, 054 (2003) arXiv:hep-th/0210209.

[3] A. Flournoy, B. Wecht and B. Williams, arXiv:hep-th/0404217.

[4] S. Kachru, M. B. Schulz, P. K. Tripathy and S. P. Trivedi, JHEP 0303, 061 (2003) arXiv:hep-th/0211182.

[5] S. Hellerman, J. McGreevy and B. Williams, JHEP 0401, 024 (2004) arXiv:hep-th/0208174.

[6] D. A. Lowe, H. Nastase and S. Ramgoolam, Nucl. Phys. B 667 (2003) 55 arXiv:hep-th/0303173.

[7] A. Flournoy and B. Williams, arXiv:hep-th/0511126.

[8] J. Shelton, W. Taylor and B. Wecht, arXiv:hep-th/0508133.

[9] J. Gray and E. Hackett-Jones, arXiv:hep-th/0506092.

[10] C. M. Hull, JHEP 9811, 027 (1998) arXiv:hep-th/9811021.

[11] A. Strominger, S. T. Yau and E. Zaslow, Nucl. Phys. B 479, 243 (1996) arXiv:hep-th/9606040.

[12] N. Kaloper and R. C. Myers, JHEP 9905, 010 (1999) arXiv:hep-th/9901045. 
[13] C. M. Hull and R. Reid-Edwards, in preparation.

[14] A. Giveon, N. Malkin and E. Rabinovici, Phys. Lett. 238 (1990) 57.

[15] C. M. Hull and R. A. Reid-Edwards, arXiv:hep-th/0503114.

[16] J. Scherk and J. H. Schwarz, Nucl. Phys. B 153, 61 (1979).

[17] J. Maharana and J.H. Schwarz, Nucl. Phys. B390 (1993) 3 hep-th/9207016.

[18] M. Cvetic, G. W. Gibbons, H. Lu and C. N. Pope, Class. Quant. Grav. 20, 5161 (2003) arXiv:hep-th/0306043.

[19] T.H. Buscher, Phys. Lett. B194 (1987) 59; Phys. Lett. B201 (1988) 466.

[20] K. S. Narain, M. H. Sarmadi and C. Vafa, Nucl. Phys. B 288, 551 (1987).

[21] A. Dabholkar and J. A. Harvey, JHEP 9902, 006 (1999) arXiv:hep-th/9809122.

[22] T. Kugo and B. Zwiebach, Prog. Theor. Phys. 87, 801 (1992) arXiv:hep-th/9201040.

[23] M. Evans and I. Giannakis, Nucl. Phys. B 472 (1996) 139 arXiv:hep-th/9511061.

[24] R. Gregory, J. A. Harvey and G. W. Moore, Adv. Theor. Math. Phys. 1, 283 (1997) arXiv:hep-th/9708086.

[25] D. Tong, JHEP 0207, 013 (2002) arXiv:hep-th/0204186.

[26] J. A. Harvey and S. Jensen, arXiv:hep-th/0507204.

[27] K. Okuyama, JHEP 0508 (2005) 089 arXiv:hep-th/0508097.

[28] C. M. Hull and P. K. Townsend, Nucl. Phys. B 438, 109 (1995) arXiv:hep-th/9410167.

[29] A. Dabholkar and C. Hull, in preparation

[30] N. Hitchin, Quart. J. Math. Oxford Ser. 54, 281 (2003) arXiv:math.dg/0209099. 